\title{
AC 2008-773: DATA MANAGEMENT FOR OUTCOMES AND ASSESSMENT
}

Bertram Pariser, Technical Career Institute, Inc.

Cyrus Meherji, Technical Career Institute, Inc. 


\section{Data Management for Outcomes and Assessment}

\section{Abstract}

Our college faculty has been inundated with requests for data concerning Outcomes and Assessments. Often the question on most of the faculty minds is what is the purpose of keeping all this data? Will it improve the quality of education that we provide? This question is academic because Middle States and Tac Abet are very concerned and required analyses of Outcomes and Assessments.

To comply with the tide that is sweeping higher education I have selected Microsoft Access as a starting point. In each class on the first day I set up an Assess data base for the semester. The class data is stored in a table in the Data Base which is constructed with four fields: First Name, Last Name, Telephone, and email address. As the students enter the class room on the first day they are required to enter their information into the table.

The data in the table is then transferred to a Microsoft Excel work sheet. The Excel file has different worksheets. The CLASS work sheet has the basic data. The CLASS work sheet serves to record attendance. The TEST work sheet is used to recorded test results and detail tabulations of each test question are recorded. The HOMEWORK work sheet is used to record homework. By using the Assess Data base as a starting point the complex task of recording and compiling data for Outcomes and Assessments is made more manageable.

TCI The College of Technology is located in New York City Diagonally across from Penn Station. The college is a two year college and the students receive an AS and AOS degrees. The college is accredited by Middle States Accreditation, New York State Accreditation and the EET department is accredited by TAC/ABET. The student population is 3000 and is composed of inner city students and foreign students TCI has over 100 different languages spoken and makes it second in the world in diversity second only to the United Nations.

Middle States Commission on Higher Education and the Technology Accreditation Commission of the Accreditation Board of Engineering and Technology (TAC/ABET) are very concerned and require Outcomes and Assessments at the course level as well as at the program level.

We are trying to approach Outcomes and Assessments from building a basic shell. This shell will be a database, a repository of information on the students that take a particular class. The database will contain the student's personal information. The other part of the shell will be a spreadsheet created in EXCEL that will contain basic student data imported from ACCESS, test scores, attendance and homework. Each of the above categories will be a separate sheet in the EXCEL spreadsheet.

Primarily, we are going to build this shell and have the students populate the database with their personal information. Therefore, we will be concentrating on data collection in this paper. Data that will be collected and organized in this shell environment. 
Microsoft ACCESS was selected as a starting point to gather data. In each class on the first day, I set up an ACCESS database for the semester. The class data is stored in a table in the database which is constructed with four fields:

- $\quad$ FIRST Name

- LAST Name

- TELEPHONE

- EMAIL address.

As the students enter the class room on the first day they are requested to enter their information into this table. The data in the table is then transferred to a Microsoft EXCEL worksheet. The EXCEL file has different worksheets. .

\begin{tabular}{|l|l|l|}
\hline WORK SHEET \# & NAME & DATA \\
\hline 1 & CLASS & $\begin{array}{l}\text { ATTENDANCE POPULATED } \\
\text { BY STUDENTS }\end{array}$ \\
\hline 2 & TESTS & TESTS \\
\hline 3 & HOMEWORK & HOMEWORK \\
\hline
\end{tabular}

By using the ACCESS database as a starting point the complex task of recording and compiling data for Outcomes and Assessments is made more manageable.

We begin our data collection by designing an ACCESS database

To design an ACCESS database

Create a folder on the Desktop and call it ASEE

1. Start $>$ All Programs $>$ Microsoft $>$ Microsoft Office

2. Select Microsoft ACCESS 2007

3. Select New Blank database

4. Save As with filename ASEE 2008 in the ASEE folder on the desktop.

5. Click Create 
6. Click on the Views tab

7. Click on Design Views

8. Name the table PHY102 in the Save As box.

9. The field name in the first row is ID

10. ID field has a data type of Auto Number

11. Click on the second row

12. Type FIRST in the field name

13. Press Tab

14. In the data type TEXT is selected

15. Under the General tab Field Size should be made 20.

16. Click on the third row

17. In the field name enter LAST for last name

18. Press Tab

19. Under the General tab Field Size should be made 20

20. Click on the fourth row. Enter TELEPHONE in that field.

21. In the data type TEXT is selected on the bottom enter 18 in the field size

22. Under the General tab click on the box to the right of the Input mask and click on the buil button

23. A message asks you to save the data first, click yes

24. Select phone number and click next

25. Click Next again

26. How do you want to store the data selected with the symbols in the mask. Click NEXT, click FINISH.

27. On the next row enter EMAIL in the field name

28. Data type is text and the field size in the General tab is 50

29. Click the box to the left of the Field Name to select the entered data 


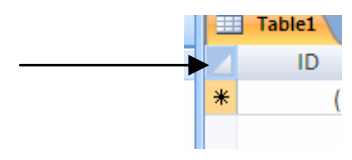

30. Right click on the selected area and select copy

31. On the top tab click Create and then click Table twice

32. Click view and select design view

33. Save the table as PHY 201

34. Move the mouse to the Second Row and right click paste

35. The second row is highlighted on the top click Delete Rows on the message click Yes, Click Save

36. Repeat the same process for each class.

Populating the Databases.

On the first day of class open the database and ask each student to enter his own data in the four fields FIRST, LAST, TELEPHONE and EMAIL. Students like to do this hands-on exercise and make sure that they Capitalize the first letter of their names. Some of the field values might not fit in the specified width of the columns. Adjust the column widths as needed.

After the data base is populated we use the data to make and MS-EXCEL grading document. The first step in making an EXCEL grading document is to sort the access table alphabetically. This is necessary since data when it is entered has been tracked by an index number and not alphabetically.

To design an EXCEL grading Document

1. Open the ACCESS database and select the first table PHY102

2. Click the column LAST and highlight the entire column.

3. In the tab labeled Home, select Sort \& Filter

4. Click the AZ Down arrow (Ascending Sort) and this will sort the database alphabetically.

5. Click the triangle $\longrightarrow *{ }_{*}^{10}$ under PHY102 at the left of the table to highlight the table

6. Move the mouse to the bottom row of the table and right click 
7. Select copy

8. Now open MS-EXCEL 2007

9. Highlight Columns A,B,C,D,E,\&F

10. Right Click

11. Select Column Width

12. Enter 50 and click $\mathrm{OK}$

13. Click cell A1

14. Right Click and select Paste

15. On the home tab select Cells, Format cells and click

16. Select Auto-Fit Column Width

17. On the bottom click on the tab labeled Sheet 1

18. Select Rename and enter PHY102

19. Press Enter

20. Highlight Columns FIRST and LAST

21. Right click, select copy. The border of the columns should be blinking

22. Click the Sheet 2 tab at the bottom

23. Highlight columns A and B on sheet 2

24. Right click column width and select 50

25. Right click in cell A1 and select paste

26. On the Home tab select Format and Auto-Fit column width

27. Right click on Sheet 2 tab at the bottom, Rename it to Attendance

28. Repeat steps 22 to 27 again and Rename this tab to Tests

29. Click on the Office button and click on Save As

30. Save this EXCEL file as PHY102Grades in the ASEE folder on the Desktop. 
31. Repeat this for all the other courses that you are teaching.

\section{Grading}

TCI's EET Electronic Engineering Technology Department is accredited by TAC-ABET. Each semester we complete a Course Objectives and Assessment Techniques matrix to see the students' progress.

In grading an exam each question is worth 10 points. If the solution is correct the grade is 10 . If the solution is blank the grade is 0 . If the solution has some merit the grade is between 0 and 10 . The test grading is done in the following manner. We grade Question 1 on each test, then Question 2 on each test and so on. In this manner we are able to see qualitatively from the student answers if the material has been absorbed by them. This assures that the subjected assessment is uniform for each student.

Below in Figure 1 we have shown a quantitative assesment. We are using the test scores from a physics test. This test was graded as described above.

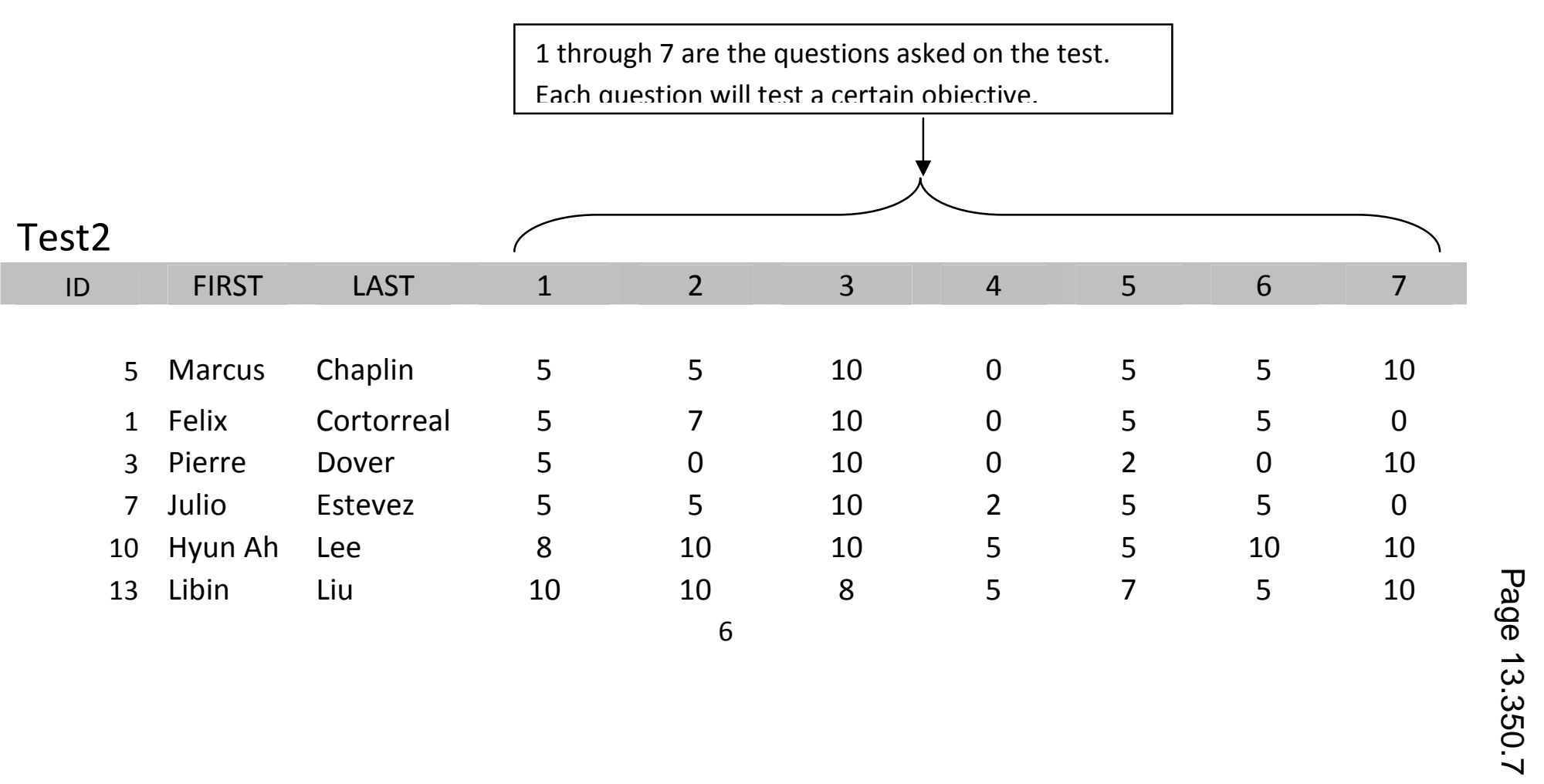




$\begin{array}{rllccccccc}6 & \text { Marlon } & \text { Lizardo } & 5 & 10 & 10 & 5 & 5 & 5 & 0 \\ 4 & \text { Tarik } & \text { Saab } & 5 & 5 & 8 & 2 & 8 & 10 & 10 \\ 11 & \text { Jonathan } & \text { Salas } & 0 & 0 & 10 & 0 & 0 & 0 & 10 \\ 2 & \text { Rohan } & \text { Surajpal } & 5 & 5 & 10 & 0 & 5 & 5 & 0 \\ 15 & \text { Bosco } & \text { Yip } & 10 & 5 & 10 & 5 & 7 & 5 & 0 \\ 12 & \text { Ripley } & \text { Young } & 0 & 5 & 7 & 0 & 2 & 0 & 10 \\ \text { rage } & & & & & & & & \\ \text { ctive } & & 5.25 & 5.58 & 9.42 & 2.00 & 4.67 & 4.58 & 5.83 \\ & & 83 \% & 83 \% & 100 \% & 33 \% & 75 \% & 75 \% & 58 \%\end{array}$

Figure 1

Each question (1 through 7) in Figure 1 is worth 10 points. If we take the average of the points awarded for all students for question 1 we find the average is 5.25. The alternate method is that we pick a cutoff score 5 and say that we believe that the student has reached the Objective. This alternate method quantizes a qualitative view of the reaching the objective. In this case 10 students out of 12 met the objective or $83 \%$ met the objective.

Figure 2 AVERAGE GRADE FOR EACH QUESTION shows us that the objective might not have been fully understood since on the average 5.25 is higher than the cutoff score of 5. However, on the other hand we see Figure 3 STUDENTS REACHING THE OBJECTIVE FOR EACH QUESTION that $83 \%$ of the people have actually understood the objective. We believe that both these measures are necessary and useful.

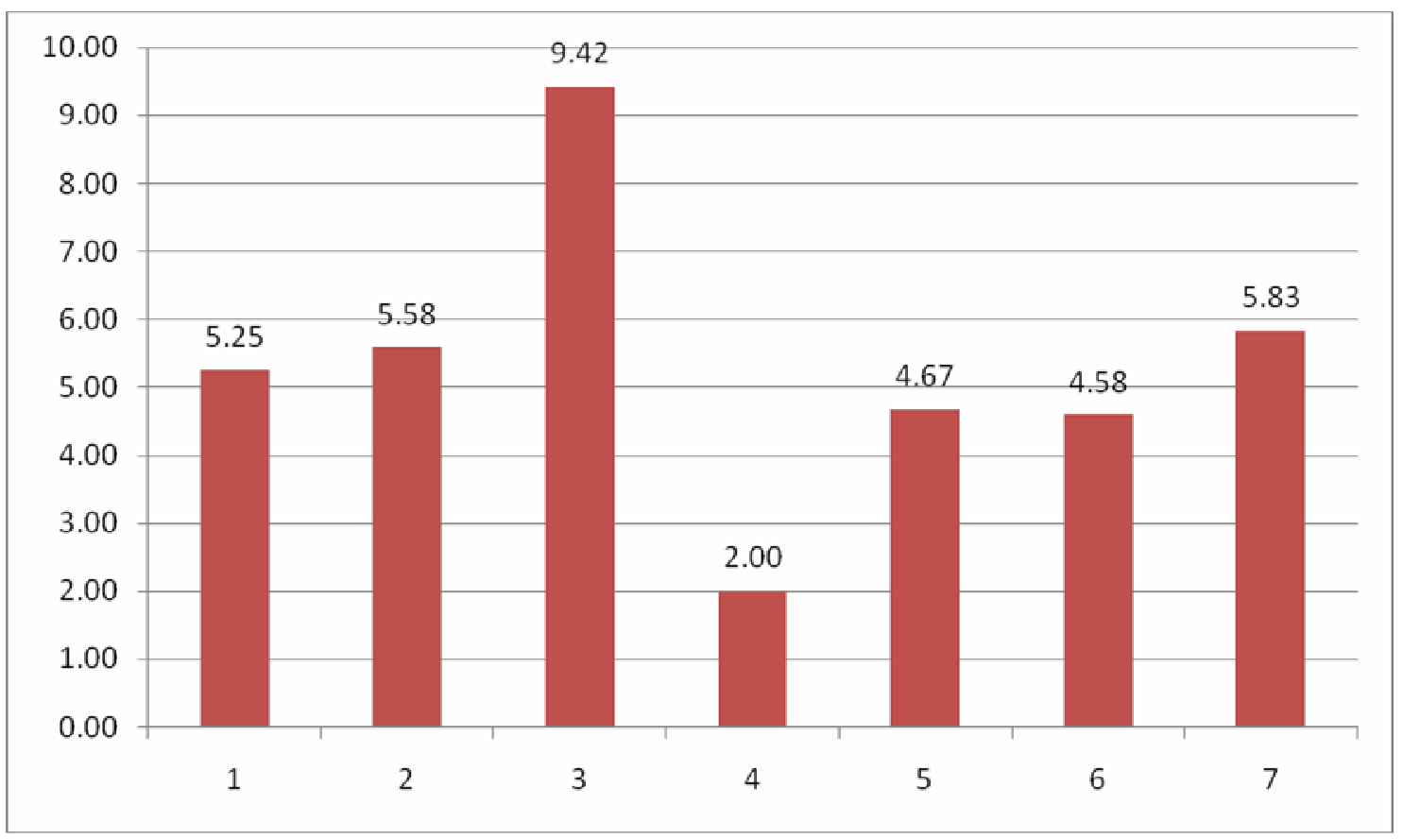


Figure 2 AVERAGE GRADE FOR EACH QUESTION

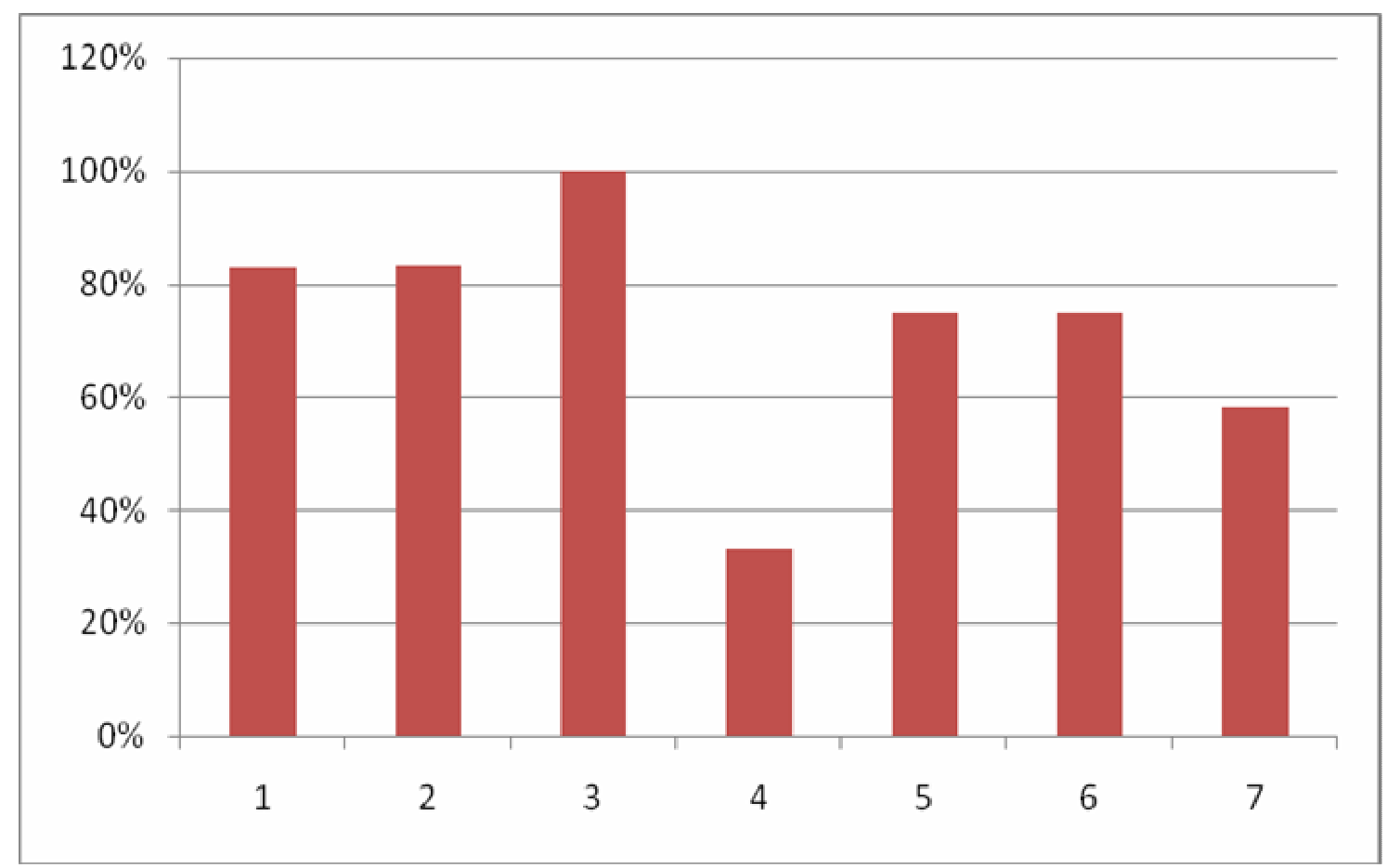

Figure 3 STUDENTS REACHING THE OBJECTIVE FOR EACH QUESTION

\section{Conclusion}

By using the Microsoft Access Data Base as a starting document and taking that information and producing a Microsoft Excel Grading Sheet the data collection process is systematized and functional and easy to use. Producing meaningfully is the starting point to analyzing Outcomes and Assessment.

\section{$\underline{\text { AUTHORS }}$}

Dr. Bertram Pariser is a faculty member in the Electronics Engineering Technology program at TCI, The College for Technology. He has published 23 papers and serves as the Faculty Advisor to Tau Alpha Pi National Honor Society. He Co-Founded 5 venture companies, and as a management consultant successfully catalyzed over $\$ 100$ million of new shareholder value in client businesses. He has a Ph.D. from Columbia University in Electrical Engineering \& Plasma Physics, and BS in Electrical Engineering from MIT. Bert.pariser@tcicollege.edu 
Cyrus Meherji is a faculty member in the Electronics Engineering Technology and Information Technology Departments. His primary responsibilities are teaching computer programming and network infrastructure. cmeherji@tcicollege.edu 